\title{
Varieties of general type with the same Betti numbers as $\mathbb{P}^{1} \times \mathbb{P}^{1} \times \cdots \times \mathbb{P}^{1}$
}

\author{
AMIR DŽAMBIĆ
}

\begin{abstract}
We study quotients $\Gamma \backslash \mathbb{H}^{n}$ of the $n$-fold product of the upper half-plane $\mathbb{H}$ by irreducible and torsion-free lattices $\Gamma<\mathrm{PSL}_{2}(\mathbb{R})^{n}$ with the same Betti numbers as the $n$-fold product $\left(\mathbb{P}^{1}\right)^{n}$ of projective lines. Such varieties are called fake products of projective lines or fake $\left(\mathbb{P}^{1}\right)^{n}$. These are higher-dimensional analogs of fake quadrics. In this paper we show that the number of fake $\left(\mathbb{P}^{1}\right)^{n}$ is finite (independently of $n$ ), we give examples of fake $\left(\mathbb{P}^{1}\right)^{4}$ and show that for $n>4$ there are no fake $\left(\mathbb{P}^{1}\right)^{n}$ of the form $\Gamma \backslash \mathbb{H}^{n}$ with $\Gamma$ contained in the norm-one group of a maximal order of a quaternion algebra over a real number field.
\end{abstract}

11F06, 22E40

\section{Introduction}

After their classification of fake projective planes (see Prasad and Yeung $[15 ; 17]$ ) and the study of arithmetic fake projective spaces and fake Grassmannians (see [16]), in their paper [18], G Prasad and S-K Yeung introduced the general notion of a fake compact hermitian symmetric space and studied these spaces in detail. Let $X=G / K$ be a hermitian symmetric space of noncompact type and $\hat{X}$ the compact dual of $X$. Then, by definition, a quotient $X_{\Gamma}=\Gamma \backslash X$ of $X$ by a cocompact and torsion-free discrete subgroup is a fake compact hermitian symmetric space or fake $\hat{X}$ if $X_{\Gamma}$ has the same Betti numbers as $\hat{X}$; such a fake $\hat{X}$ is called irreducible, resp. arithmetic, if $\Gamma$ is irreducible, resp. arithmetic. One of the main results in Prasad and Yeung [18] is that there are no compact irreducible arithmetic fake hermitian symmetric spaces of type other than $A_{n}$ with $n \leq 4$. Here, the type of $X_{\Gamma}$ refers to the type of the irreducible factors $X_{i}$ of the universal covering $X=X_{1} \times \cdots \times X_{S}$ of $X_{\Gamma}$ according to Cartan's classification of irreducible hermitian symmetric spaces of noncompact type. Two-dimensional fake $A_{2}$ are exactly the fake projective planes, which are completely classified. In Prasad and Yeung [16] we find examples of fourdimensional fake $A_{2}$, that is, fake products of projective planes $\mathbb{P}^{2} \times \mathbb{P}^{2}$. Note that the case of fake $A_{1}$ is not covered in [18]. By definition, a compact fake $A_{1}$ of dimension $n$ (called $n$-dimensional fake product of projective lines in sequel) is a 
compact quotient $\Gamma \backslash \mathbb{H}^{n}$ of the product of $n$ copies of the complex upper half-plane $\mathbb{H}$ by a cocompact torsion-free lattice $\Gamma \subset \mathrm{PSL}_{2}(\mathbb{R})^{n}$ with the same Betti numbers as the product $\mathbb{P}^{1} \times \cdots \times \mathbb{P}^{1}$ of $n$ copies of the complex projective line $\mathbb{P}^{1}=\mathbb{P}^{1}(\mathbb{C})$. The notion of an $n$-dimensional compact fake $A_{1}$ is meaningful only for $n \geq 2$ and in this case an irreducible fake $A_{1}$ is automatically arithmetic. Two-dimensional fake $A_{1}$ are also known as fake quadrics; see Hirzebruch [10, page 779f]. There are many known irreducible as well as nonirreducible fake quadrics (see for instance Shavel [19] or the author [8] for the irreducible case and Bauer, Catanese and Grunewald [1] for the nonirreducible case). It is known that no fake products of projective lines of odd dimension are possible. In this note we study the existence questions of irreducible compact fake products of projective lines:

Theorem A (See Theorem 3.2) There exists a constant $c>0$ such that for any integer $n>c$ there exists no irreducible fake $\left(\mathbb{P}^{1}\right)^{n}$. The number of fake $\left(\mathbb{P}^{1}\right)^{n}$ is finite.

Moreover we discuss the existence of irreducible fake products of projective lines with the fundamental group contained in the norm-one group of a maximal order and show:

Theorem B (See Lemma 3.4 and Theorems 3.5, 4.5, 4.2 and 4.4) There exist two nonisomorphic 4-dimensional irreducible fake products of projective lines of the form $\Gamma \backslash \mathbb{H}^{4}$, where $\Gamma$ is the norm-one group of a maximal order in totally indefinite quaternion algebras over the maximal totally real subfields of cyclotomic fields $\mathbb{Q}\left(\xi_{20}\right)$ and $\mathbb{Q}\left(\xi_{24}\right)$ of $20^{\text {th }}$ and $24^{\text {th }}$ roots of unity. Moreover, fake products of projective lines whose fundamental group is contained in a norm-one group of a maximal order exist only in dimension 2 and 4 . In dimension 4, the examples above are the only such examples up to isomorphism.

Acknowledgments The author would like to thank Sai-Kee Yeung and Mikhail Belolipetsky for the helpful comments and discussions on the subject.

\section{Irreducible quotients of the polydisc}

Let $\mathbb{H}$ denote the complex upper half-plane and $\mathbb{H}^{n}=\mathbb{H} \times \cdots \times \mathbb{H}$ the product of $n$ copies of $\mathbb{H}$. Since $\mathbb{H}$ is biholomorphically equivalent to the unit disc $\mathbb{D} \subset \mathbb{C}$, we will use the short term polydisc to name $\mathbb{H}^{n}$. The group $\mathrm{GL}_{2}^{+}(\mathbb{R})^{n}=\mathrm{GL}_{2}^{+}(\mathbb{R}) \times$ $\cdots \times \mathrm{GL}_{2}^{+}(\mathbb{R})$, with $\mathrm{GL}_{2}^{+}(\mathbb{R})$ the group of $2 \times 2$-matrices with positive determinant, acts on $\mathbb{H}^{n}$ componentwise as a group of linear fractional transformations. The quotient $\mathrm{PGL}_{2}^{+}(\mathbb{R})^{n}=\left(\mathrm{GL}_{2}^{+}(\mathbb{R}) / \mathbb{R}^{*}\right)^{n} \cong \mathrm{PSL}_{2}(\mathbb{R})^{n}$ is identified with Aut ${ }^{+}\left(\mathbb{H}^{n}\right)$, the group of biholomorphic automorphisms which preserve each factor. A lattice 
$\Gamma \subset$ Aut $^{+}\left(\mathbb{H}^{n}\right)$, that is, a discrete subgroup of finite covolume, is called irreducible if $\Gamma$ is not commensurable with a product $\Gamma_{1} \times \Gamma_{2}$ of two nontrivial lattices $\Gamma_{1} \subset$ Aut $^{+}\left(\mathbb{H}^{r}\right)$, $\Gamma_{2} \in \mathrm{Aut}^{+}\left(\mathbb{H}^{n-r}\right)$ for some $0<r<n$. For $n \geq 2$, irreducible lattices can be constructed arithmetically in the following way.

Let $k$ be a totally real number field of degree $m=[k: \mathbb{Q}]$ and let $A$ be a quaternion algebra over $k$. Choosing an ordering $v_{\infty, 1}, \ldots, v_{\infty, m}$ of the infinite places of $k$, assume that $A$ is unramified at first $n \leq m$ places $v_{\infty, 1}, \ldots, v_{\infty, n}$ and ramified at the remaining infinite places $v_{\infty, n+1}, \ldots, v_{\infty, m}$. Additionally assume that $A$ ramifies at the finite places $\mathfrak{p}_{1}, \ldots, \mathfrak{p}_{r}$ of $k$. Let $d_{A}=\prod_{i=1}^{r} \mathfrak{p}_{i}$ denote the reduced discriminant of $A$. Then $m-n+r \equiv 0 \bmod 2$ and, under this condition, $A$ is uniquely determined by the choice of $n$ unramified infinite places and the reduced discriminant $d_{A}$ up to isomorphism. We will write $A=A\left(k ; m, n, d_{A}\right)=A\left(k ; m, n, \mathfrak{p}_{1}, \ldots, \mathfrak{p}_{r}\right)$ to denote the isomorphism class of such $A$. If $\mathcal{O} \subset A$ is an order in $A$ then, under the embeddings $A \hookrightarrow A \otimes_{k} k_{v_{\infty, i}} \cong M_{2}(\mathbb{R})$ for $i=1, \ldots, n$, the group $\mathcal{O}^{+}=\{x \in \mathcal{O} \mid \operatorname{Nrd}(x)$ is totally positive unit in $k\}$ becomes a subgroup of $\mathrm{GL}_{2}^{+}(\mathbb{R})^{n}$, where $\mathrm{Nrd}$ denotes the reduced norm. The group $\Gamma_{\mathcal{O}}^{+}=\mathcal{O}^{+} / k^{*}$ is a lattice in $\operatorname{Aut}^{+}(\mathbb{H})^{n}$ and, moreover, $\Gamma_{\mathcal{O}}^{+}$is an irreducible lattice and so is every $\Gamma \subset \operatorname{Aut}^{+}\left(\mathbb{H}^{n}\right)$ which is commensurable with $\Gamma_{\mathcal{O}}^{+}$. We say that $\Gamma \subset \operatorname{Aut}^{+}\left(\mathbb{H}^{n}\right)$ is an arithmetic lattice if there exits a number field $k$, a quaternion algebra $A=A\left(k ; m, n, d_{A}\right)$ and a maximal order $\mathcal{O}$ in $A$ such that $\Gamma$ is commensurable with $\Gamma_{\mathcal{O}}^{+}$. For example $\Gamma_{\mathcal{O}}^{1}=\{x \in \mathcal{O} \mid \operatorname{Nrd}(x)=1\} /\{ \pm 1\}$ or any congruence subgroup in $\Gamma_{\mathcal{O}}^{+}$or $\Gamma_{\mathcal{O}}^{1}$ is, by definition, arithmetic. By the celebrated theorem of Margulis, for $n \geq 2$, every irreducible lattice $\Gamma \in \mathrm{Aut}^{+}(\mathbb{H})^{n}$ is an arithmetic lattice.

Let $\Gamma$ be an irreducible lattice in $\operatorname{Aut}^{+}\left(\mathbb{H}^{n}\right)$ commensurable with $\Gamma_{\mathcal{O}}^{+}$, where $\mathcal{O}$ is a maximal order in the quaternion algebra $A=A\left(k ; m, n, d_{A}\right)$. As a corollary to Godement's compactness criterion we have that $\Gamma$ is a cocompact lattice in $\operatorname{Aut}^{+}\left(\mathbb{H}^{n}\right)$ if and only if $A$ is a division algebra, or equivalently if and only if $\Gamma$ is not commensurable with a Hilbert modular group. Let $X_{\Gamma}=\Gamma \backslash \mathbb{H}^{n}$ be the orbit space under the action of $\Gamma$ on $\mathbb{H}^{n}$. Then $X_{\Gamma}$ is a compact locally symmetric space. If $\Gamma$ is torsion free, $X_{\Gamma}$ is an $n$-dimensional complex manifold and even more, in this case $X_{\Gamma}$ is a smooth projective variety whose canonical line bundle is ample. In particular, for torsion-free and cocompact lattices $\Gamma, X_{\Gamma}$ is a variety of general type.

By Hirzebruch's proportionality theorem, numerical invariants of $X_{\Gamma}$ are closely related to the numerical invariants of the compact dual $\left(\mathbb{P}^{1}\right)^{n}$ of $\mathbb{H}^{n}$ (see Hirzebruch [9]). In fact, only the knowledge of the Euler number $e\left(X_{\Gamma}\right)$ determines the complete Hodge diamond of $X_{\Gamma}$ (see Matsushima and Shimura [12] or Lemma 2.4 below). On the other hand, the Euler number can be computed by a volume formula which generalizes Siegel's formula for the volume of the fundamental domain of $\mathrm{SL}_{2}(\mathbb{Z})$ in 
terms of Riemann zeta function. Namely, consider the $\operatorname{Aut}^{+}(\mathbb{H})^{n}$-invariant volume form $v=(-2 \pi)^{-n} \prod_{1 \leq i \leq n} d x_{i} \wedge d y_{i} / y_{i}^{2}$ on $\mathbb{H}^{n}$. Then for torsion-free cocompact lattices $\Gamma \subset$ Aut $^{+}\left(\mathbb{H}^{n}\right)$ we have $e\left(X_{\Gamma}\right)=\operatorname{vol}\left(X_{\Gamma}\right)$, where $\operatorname{vol}\left(X_{\Gamma}\right)$ denotes the volume of a $\Gamma$-fundamental domain in $\mathbb{H}^{n}$ with respect to $v$.

Lemma 2.1 (Vignéras [22]) Let $k$ be a totally real number field of degree $m=[k: \mathbb{Q}]$ and let $A=A\left(k ; m, n, d_{A}\right)$ be a quaternion algebra over $k$. Fix a maximal order $\mathcal{O} \subset A$ and let $\Gamma \subset \mathrm{PGL}_{2}^{+}(\mathbb{R})^{n}$ be a subgroup commensurable with $\Gamma_{\mathcal{O}}^{1}=\mathcal{O}^{1} / \pm 1$. Then

$$
\operatorname{vol}\left(X_{\Gamma}\right)=\left[\Gamma_{\mathcal{O}}^{1}: \Gamma\right](-1)^{m+n} 2^{n-m+1} \zeta_{k}(-1) \prod_{\mathfrak{p} \mid d_{A}}(N \mathfrak{p}-1),
$$

where $\left[\Gamma_{\mathcal{O}}^{1}: \Gamma\right]=\left[\Gamma_{\mathcal{O}}^{1}: \Gamma \cap \Gamma_{\mathcal{O}}^{1}\right] /\left[\Gamma: \Gamma \cap \Gamma_{\mathcal{O}}^{1}\right]$ denotes the generalized index between $\Gamma$ and $\Gamma_{\mathcal{O}}^{1}, \zeta_{k}(\cdot)$ is the Dedekind zeta function and where for an integral prime ideal $\mathfrak{p}$ in $k, N \mathfrak{p}=\left|\mathcal{O}_{k} / \mathfrak{p}\right|$ is the norm of $\mathfrak{p}$.

\subsection{Fake products of projective lines whose universal covering is the polydisc}

Let $\Gamma$ be a torsion-free cocompact irreducible lattice in $\operatorname{Aut}^{+}\left(\mathbb{H}^{n}\right)$. As mentioned above, the quotient $X_{\Gamma}=\Gamma \backslash \mathbb{H}^{n}$ is an $n$-dimensional smooth projective variety of general type whose Betti numbers are closely related to the Betti numbers of $\left(\mathbb{P}^{1}\right)^{n}$.

Definition 2.2 We say that a compact quotient $X_{\Gamma}$ of the $n$-dimensional polydisc $\mathbb{H}^{n}$ is a compact fake product of projective lines (or simply fake $\left.\left(\mathbb{P}^{1}\right)^{n}\right)$ if $\Gamma$ is a cocompact and torsion-free lattice in Aut $^{+}\left(\mathbb{H}^{n}\right)$ and $X_{\Gamma}=\Gamma \backslash \mathbb{H}^{n}$ has the same Betti numbers as $\left(\mathbb{P}^{1}\right)^{n}$. We say that $X_{\Gamma}$ is irreducible if $\Gamma$ is an irreducible lattice in $\operatorname{Aut}^{+}\left(\mathbb{H}^{n}\right)$.

Remark 2.3 (1) As already mentioned in the introduction, many examples of irreducible and nonirreducible fake $\mathbb{P}^{1} \times \mathbb{P}^{1}$ (fake quadrics) are known. The nonirreducible fake quadrics are examples of so-called Beauville surfaces. In higher dimensions, excluding the "trivial" cases of products of fake quadrics, no examples of fake $\left(\mathbb{P}^{1}\right)^{n}$ seem to be known. It is an open question to construct such higher-dimensional fake $\left(\mathbb{P}^{1}\right)^{n}$ which are not products of fake $\left(\mathbb{P}^{1}\right)^{n}$ in lower dimension. In this paper we will concentrate on irreducible fake $\left(\mathbb{P}^{1}\right)^{n}$. But other "nontrivial" constructions are to be analyzed. In particular, this includes the construction of higher-dimensional varieties isogenous to a product of curves (see Catanese [6]) with given Betti numbers which are not products of Beauville surfaces.

(2) More generally, we could skip the condition on the universal covering, and define a fake $\left(\mathbb{P}^{1}\right)^{n}$ as a variety of general type with the same Betti numbers as $\left(\mathbb{P}^{1}\right)^{n}$. Here 
an open question is to identify the universal covering of such a variety; is the universal covering of a variety of general type with the same Betti numbers as $\left(\mathbb{P}^{1}\right)^{n}$ always $\mathbb{H}^{n}$ (compare [10, page 780])?

A theorem of Matsushima and Shimura gives a characterization of irreducible fake products of projective lines:

Lemma 2.4 [12, Theorems 7.2, 7.3] Let $\Gamma$ be an irreducible and torsion-free lattice in $\mathrm{Aut}^{+}\left(\mathbb{H}^{n}\right)$ and let $b_{i}\left(X_{\Gamma}\right)$ denote the $i^{\text {th }}$ Betti number of $X_{\Gamma}$. Then

- for $i \neq n, b_{i}\left(X_{\Gamma}\right)=b_{i}\left(\left(\mathbb{P}^{1}\right)^{n}\right)$;

- $b_{n}\left(X_{\Gamma}\right)=b_{n}\left(\left(\mathbb{P}^{1}\right)^{n}\right)+2^{n} h^{n, 0}\left(X_{\Gamma}\right)$, where $h^{n, 0}\left(X_{\Gamma}\right)=\operatorname{dim} H^{0}\left(X_{\Gamma}, \Omega_{X_{\Gamma}}^{n}\right)$.

If $n=\operatorname{dim} X_{\Gamma}$ is odd, $X_{\Gamma}$ cannot be a fake product of projective lines. For $n$ even, $X_{\Gamma}$ is a fake product of projective lines if and only if the arithmetic genus $\chi\left(X_{\Gamma}\right)$ equals 1 .

Since only $b_{n}\left(X_{\Gamma}\right)$ may be different from $b_{n}\left(\left(\mathbb{P}^{1}\right)^{n}\right)$, we can characterize fake products of projective lines also by the value of the Euler number $e\left(X_{\Gamma}\right)$.

Corollary 2.5 For even $n$, a quotient $X_{\Gamma}$ is a fake $\left(\mathbb{P}^{1}\right)^{n}$ if and only if $e\left(X_{\Gamma}\right)=$ $e\left(\left(\mathbb{P}^{1}\right)^{n}\right)=2^{n}$.

\section{Finiteness results on irreducible fake $\left(\mathbb{P}^{1}\right)^{n}$}

In this section we will prove the finiteness result which states that the dimension of a fake $\left(\mathbb{P}^{1}\right)^{n}$ is bounded by an absolute constant from above and that in each dimension there are only finitely many fake $\left(\mathbb{P}^{1}\right)^{n}$. Moreover we will prove the nonexistence of irreducible fake $\left(\mathbb{P}^{1}\right)^{n} X_{\Gamma}$ whose fundamental group $\Gamma$ is contained in a norm-one group $\Gamma_{\mathcal{O}}^{1}$ of maximal order of a quaternion algebra over a real number field in all dimensions $n>4$. In fact, we will list all the isomorphism classes of quaternion algebras which contain a torsion-free group $\Gamma \leq \Gamma_{\mathcal{O}}^{1}$ such that $X_{\Gamma}$ is a 4-dimensional fake product of projective lines.

Recall that the fundamental group $\Gamma$ of an irreducible $n$-dimensional fake product of projective lines is an arithmetic lattice commensurable with $\Gamma_{\mathcal{O}}^{1}$, where $\mathcal{O}$ is a maximal order in a quaternion algebra $A=A\left(k ; m, n, d_{A}\right)$ over a totally real number field $k$ of degree $m$ with prescribed ramification at infinite places. If $\Gamma$ is contained in a lattice $\Delta$ we have $\operatorname{vol}\left(X_{\Delta}\right) \leq \operatorname{vol}\left(X_{\Gamma}\right)=e\left(X_{\Gamma}\right)=2^{n}$. Every $\Gamma$ is contained in a maximal lattice $\Delta$ with finite index, and in the first step we will show that for $n>c$ (where $c$ is a constant) every maximal irreducible lattice $\Delta<\operatorname{PSL}_{2}(\mathbb{R})^{n}$ satisfies $\operatorname{vol}(\Delta)>2^{n}$. 
In the second step we shall show that in a fixed dimension $n$ there exist only finitely many conjugacy classes of maximal (irreducible) lattices $\Delta$ in $\operatorname{PSL}_{2}(\mathbb{R})^{n}$ such that $\operatorname{vol}(\Delta) \leq 2^{n}$. For this purpose we will intensively make use of results in Borel [4] and Chinburg and Friedman [7] where volumes and commensurabilities between arithmetic lattices of $\mathrm{PGL}_{2}(\mathbb{R})^{a} \times \mathrm{PGL}_{2}(\mathbb{C})^{b}$ are studied in great detail. The most relevant results for our purposes are summarized in the following.

Lemma 3.1 Let $k$ be totally real number field of degree $m$ and let

$$
A=A\left(k ; m, n, \mathfrak{p}_{1}, \ldots, \mathfrak{p}_{r}\right)
$$

be a quaternion algebra over $k$ with $m \geq n \geq 1$ (that is, $A$ satisfies the Eichler condition).

(1) The maximal arithmetic subgroup of

$$
A^{+} / k^{*}=\{x \in A \mid \operatorname{Nrd}(x) \text { is totally positive }\} / k^{*}
$$

which contains $\Gamma_{\mathcal{O}}^{1}$ for a maximal order $\mathcal{O}$ in $A$ is $N \Gamma_{\mathcal{O}}^{+}=\left\{x \in A^{+} \mid x \mathcal{O} x^{-1}=\mathcal{O}\right\} / k^{*}$; compare [4, 4.9, 8.4-8.6; 7, Lemma 2.1]. The index of $\Gamma_{\mathcal{O}}^{1}$ in $N \Gamma_{\mathcal{O}}^{+}$is $\left[N \Gamma_{\mathcal{O}}^{+}: \Gamma_{\mathcal{O}}^{1}\right]=$ $2^{r}\left[k_{A}: k\right]$, where $k_{A}$ is the maximal abelian extension of $k$ which is unramified at all finite places of $k$ and such that its Galois group $\operatorname{Gal}\left(k_{A} / k\right)$ is elementary 2-abelian and in which all the prime ideals $\mathfrak{p}_{1}, \ldots, \mathfrak{p}_{r}$ are totally split.

(2) Let $S$ be a finite set of primes of $k$ such that $\mathfrak{p}_{i} \notin S$ for $i=1, \ldots, r$ and let $\mathcal{O}(S)$ be the Eichler order of level $\prod_{\mathfrak{q} \in S} \mathfrak{q}$. Let $\Gamma_{S, \mathcal{O}}^{+}$be the normalizer of $\mathcal{O}(S)$ in $A^{+} / k^{*}$. Then every maximal arithmetic subgroup of $A^{+} / k^{*}$ is (a conjugate of) a lattice of the form $\Gamma_{S, \mathcal{O}}^{+}$with $\Gamma_{\varnothing, \mathcal{O}}^{+}=N \Gamma_{\mathcal{O}^{\prime}}^{+}$for some maximal order $\mathcal{O}^{\prime}$. There exists an integer $0 \leq s \leq|S|$ such that the generalized index $\left[N \Gamma_{\mathcal{O}}^{+}: \Gamma_{S, \mathcal{O}}^{+}\right]$equals $2^{-s} \prod_{\mathfrak{q} \in S}(N \mathfrak{q}+1)$. For any $S$ we have $\operatorname{vol}\left(X_{N \Gamma_{\mathcal{O}}^{+}}\right) \leq \operatorname{vol}\left(\Gamma_{S, \mathcal{O}}^{+}\right)$with equality if and only if $S=\varnothing$. There exist only finitely many conjugacy classes of maximal lattices $\Gamma_{S, \mathcal{O}}^{+}$commensurable with a given $N \Gamma_{\mathcal{O}}^{+}$such that $\left[N \Gamma_{\mathcal{O}}^{+}: \Gamma_{S, \mathcal{O}}^{+}\right] \leq c$ for any given $c>0$; compare $[4,4.4$, 5.3] and Maclachlan and Reid [11, 11.4].

(3) Let $\mathcal{C}(k, A)$ be the set of all irreducible lattices in $\operatorname{PSL}_{2}(\mathbb{R})^{n}$ which are commensurable with $\Gamma_{\mathcal{O}}^{1}$ for some maximal order $\mathcal{O}$ in $A / k$. Then the function $\Gamma \mapsto \operatorname{vol}\left(X_{\Gamma}\right)$ takes its minimum on a conjugate of $N \Gamma_{\mathcal{O}}^{+}$. Let $e$ be the number of dyadic places of $k$ (finite places lying over 2 ) not dividing the reduced discriminant of $A$. Then $\operatorname{vol}(\Gamma)$ is a positive integral multiple of $2^{-e} \operatorname{vol}\left(X_{N \Gamma_{\mathcal{O}}^{+}}\right)$for any $\Gamma$ in $\mathcal{C}(k, A)$; compare $[4,5.4]$.

The finiteness of the set of irreducible fake $\left(\mathbb{P}^{1}\right)^{n}$ follows immediately from the following theorem. 
Theorem 3.2 There exists a constant $c>0$ such that if $N \Gamma_{\mathcal{O}}^{+} \hookrightarrow \mathrm{PSL}_{2}(\mathbb{R})^{n}$ is a maximal lattice with $\operatorname{vol}\left(N \Gamma_{\mathcal{O}}^{+}\right) \leq 2^{n}$ then $n \leq c$. For each $n$ there are only finitely many conjugacy classes in $\mathrm{PSL}_{2}(\mathbb{R})^{n}$ of maximal lattices $\Delta$ such that $\operatorname{vol}\left(X_{\Delta}\right) \leq 2^{n}$.

Proof By Lemmas 3.1 and 2.1, the volume of $X_{N \Gamma_{\mathcal{O}}^{+}}$is given by

$$
\operatorname{vol}\left(X_{N \Gamma_{\mathcal{O}}^{+}}\right)=(-1)^{n+m} \frac{2^{n-m+1} \zeta_{k}(-1)}{2^{r}\left[k_{A}: k\right]} \prod_{i=1}^{r}\left(N \mathfrak{p}_{i}-1\right) .
$$

Let $k_{A}^{\prime}$ be the abelian extension of $k$ with the same properties as $k_{A}$ but which is additionally unramified also at all infinite places of $k$. Then (see [7, Proposition 2.1])

$$
\left[k_{A}: k\right]^{-1}=\frac{\left[\mathfrak{o}_{k}^{*}: \mathfrak{o}_{k,+}^{*}\right]}{2^{m}}\left[k_{A}^{\prime}: k\right]^{-1} .
$$

The advantage of considering $k_{A}^{\prime}$ instead of $k_{A}$ is the fact that $\left[k_{A}^{\prime}: k\right]$ divides the class number of $k$. Now recall the functional equation of the Dedekind zeta function, by which for a totally real number field $k$ of degree $m$ we have

$$
\zeta_{k}(-1)=(-1)^{m} 2^{-m} \pi^{-2 m} d_{k}^{3 / 2} \zeta_{k}(2),
$$

where $d_{k}$ is the discriminant of $k$. Keeping in mind that $n$-dimensional fake products of projective lines exist only for even $n$ we obtain

$$
\operatorname{vol}\left(X_{N \Gamma_{\mathcal{O}}^{+}}\right)=\frac{d_{k}^{3 / 2} \zeta_{k}(2)}{2^{2 m-n-1+t^{\prime}}\left[k_{A}: k\right] \pi^{2 m}} \prod_{\substack{i \in\{1, \ldots, r\} \\ N \mathfrak{p}_{i} \neq 2}} \frac{N \mathfrak{p}_{i}-1}{2},
$$

where $t^{\prime}$ is the number of those primes $\mathfrak{p}_{i}$ which are ramified in $A$ and such that $N \mathfrak{p}_{i}=2$. Let $t$ be the number of primes of $k$ which divide 2 . Then $t^{\prime} \leq t$. Define

$$
g(k, A)=\frac{d_{k}^{3 / 2} \zeta_{k}(2)}{2^{2 m-1+t} \pi^{2 m}\left[k_{A}: k\right]}=\frac{d_{k}^{3 / 2} \zeta_{k}(2)\left[\mathfrak{o}_{k}^{*}: \mathfrak{o}_{k,+}^{*}\right]}{2^{3 m-1+t} \pi^{2 m}\left[k_{A}^{\prime}: k\right]}
$$

Then

$$
\operatorname{vol}\left(X_{N \Gamma_{\mathcal{O}}^{+}}\right)=2^{t-t^{\prime}+n} g(k, A) \prod_{\substack{i \in\{1, \ldots, r\} \\ N \mathfrak{p}_{i} \neq 2}} \frac{N \mathfrak{p}_{i}-1}{2} .
$$

Assume now that $2^{n} \geq \operatorname{vol}\left(X_{N \Gamma_{\mathcal{O}}^{+}}\right)$. As $\prod_{i \in\{1, \ldots, r\} N \mathfrak{p}_{i} \neq 2} \frac{N \mathfrak{p}_{i}-1}{2} \geq 1$ and $t \geq t^{\prime}$, the inequality

$$
1 \geq g(k, A)
$$


follows. The main ingredient at this point is a lower bound for $g(k, A)$ depending on $m$ and $\left[k_{A}: k\right]$ obtained by Chinburg and Friedman. In [7, Lemma 3.2] we find the inequality

$$
g(k, A)>0.142 \exp \left(0.051 \cdot m-\frac{19.0745}{\left[k_{A}^{\prime}: k\right]}\right) .
$$

The right-hand side of (5) tends to infinity for $m \rightarrow \infty$. Thus the condition (4) implies that $m$ is bounded from above and since the dimension, or equivalently the number of unramified infinite places $n$ in $A$, satisfies $n \leq m$, then $n$ is also bounded. Now let $m$ be fixed. We want to show that in this case the discriminant of $k$ and reduced discriminant of $A$ are bounded. In order to do so, we first replace $\left[k_{A}^{\prime}: k\right]$ in (2) by the class number $h_{k}$ of $k$. Also we use the estimate on $h_{k}$ provided by the Brauer-Siegel theorem. Let $s>1$ be a real number, $k$ a totally real number field of degree $m$ with discriminant $d_{k}$, regulator $R_{k}$ and the class number $h_{k}$. Then, by the theorem of Brauer and Siegel,

$$
h_{k} R_{k} \leq 2^{1-m} s(s-1) \Gamma(s / 2)^{m}\left(\frac{d_{k}}{\pi^{m}}\right)^{s / 2} \zeta_{k}(s) .
$$

Using a lower bound for the regulator of the form $R_{k} \geq c_{1} \exp \left(c_{2} m\right)$ (see for instance Zimmert [27, page 375] for an explicit choice of the constants $c_{1}$ and $c_{2}$ ) and choosing $s=2$ in (6) we obtain an upper bound

$$
h_{k} \leq \frac{2^{2-m} d_{k} \zeta_{k}(2)}{\pi^{m} c_{1} \exp \left(c_{2} m\right)}
$$

Plugging this into (4) we obtain

$$
1 \geq g(k, A) \geq \frac{d_{k}^{3 / 2} \zeta_{k}(2)\left[\mathfrak{o}_{k}^{*}: \mathfrak{o}_{k,+}^{*}\right]}{2^{3 m-1+t} \pi^{2 m} h_{k}} \geq \frac{d_{k}^{1 / 2}\left[\mathfrak{o}_{k}^{*}: \mathfrak{o}_{k,+}^{*}\right] c_{1} \exp \left(c_{2} m\right)}{\pi^{m} 2^{2 m+t+1}} .
$$

As $\left[\mathfrak{o}_{k}^{*}: \mathfrak{o}_{k,+}^{*}\right] \geq 2$ and $t \leq m$ the above inequalities give

$$
d_{k}^{1 / 2} \leq \frac{\pi^{m} 2^{3 m}}{c_{1} \exp \left(c_{2} m\right)}
$$

For fixed $m$, the right-hand side of the last inequality is constant and it follows that the discriminant $d_{k}$ is bounded. It follows that there are only finitely many possible totally real fields which may serve as fields defining the commensurability class $\mathcal{C}(k, A)$ of $N \Gamma_{\mathcal{O}}^{+}$. If $k$ is fixed, from (3) it easily follows that if $\operatorname{vol}\left(N \Gamma_{\mathcal{O}}^{+}\right)$is bounded, the reduced discriminant $d_{A}$ is also bounded. As the reduced discriminant determines the isomorphism class of $A$ (notice that the ramification behavior at infinite places is fixed), there are only finitely many isomorphism classes of quaternion algebras which define lattices of fake $\left(\mathbb{P}^{1}\right)^{n}$. Finally, there are only finitely many nonconjugate maximal orders inside a given quaternion algebra $A$, hence only finitely many nonconjugate 
maximal lattices of type $N \Gamma_{\mathcal{O}}^{+}$inside the commensurability class $\mathcal{C}(k, A)$. It follows from Lemma 3.1(2) there are only finitely many maximal lattices $\Gamma_{S, \mathcal{O}}^{+} \in \mathcal{C}(k, A)$ with $\operatorname{vol}\left(X_{\Gamma_{S, \mathcal{O}}^{+}}\right) \leq 2^{n}$.

A natural question which arises from Theorem 3.2 is that on effectivity: is it possible to list all fake $\left(\mathbb{P}^{1}\right)^{n}$ ? Less ambitious we could ask to what extent one can make precise the bounds on invariants $n, m, d_{k}, d_{A}$ which belong to fake $\left(\mathbb{P}^{1}\right)^{n}$ ? Certainly, a careful analysis of the proof of Theorem 3.2 will provide bounds on the above invariants. For instance, (5) implies that the dimension $n$ of a fake $\left(\mathbb{P}^{1}\right)^{n}$ is less than or equal to 412 . In fact the bounds which we get are not expected to be very precise. The main hurdle is the invariant $\left[k_{A}: k\right]$ or rather $\left[k_{A}^{\prime}: k\right]$ associated with $A$, for which we apparently miss good bounds and which we are forced to estimate by the class number. Some observations on this invariant have been also made by Belolipetsky and Linowitz [3] in connection with the enumeration of fields of definition of arithmetic Kleinian reflection groups.

In order to get a first impression on how big the number of fake $\left(\mathbb{P}^{1}\right)^{n}$ can be, we will now concentrate our attention to fake $\left(\mathbb{P}^{1}\right)^{n}$ whose fundamental group is contained in the norm-one group of a maximal order. This is a class of arithmetic groups which is much more accessible than the general ones, since the critical invariant $\left[k_{A}^{\prime}: k\right]$ does not appear in the volume formula of such lattices. We will see soon that fake $\left(\mathbb{P}^{1}\right)^{n}$ with such a fundamental group are very rare. So, from now on let us assume that the lattice $\Gamma$ is contained in the norm-one group $\Gamma_{\mathcal{O}}^{1}$.

Remark 3.3 Note that the above assumption is indeed restrictive. Typical examples of lattices which are not contained in $\Gamma_{\mathcal{O}}^{1}$ are the normalizer of maximal and Eichler orders.

Under the assumption (7) the inequality $2^{n}=e\left(X_{\Gamma}\right) \geq e\left(X_{\Gamma_{\mathcal{O}}^{1}}\right)$ holds.

The functional equation (1) for the Dedekind zeta function and Lemma 2.1 imply directly the equality

$$
e\left(X_{\Gamma}\right)=2^{n} \geq e\left(X_{\Gamma_{\mathcal{O}}^{1}}\right)=2^{n-2 m+1} \pi^{-2 m} d_{k}^{3 / 2} \zeta_{k}(2) \prod_{\mathfrak{p} \mid d_{A}}(N \mathfrak{p}-1) .
$$

Next we observe that $\prod_{\mathfrak{p} \mid d_{A}}(N \mathfrak{p}-1) \geq 1$ as well as $\zeta_{k}(2)>\zeta_{\mathbb{Q}}(2 m)>1$. The last inequality $\zeta_{k}(2)>\zeta_{\mathbb{Q}}(2 m)$ (or more generally $\zeta_{k}(s)>\zeta_{\mathbb{Q}}(m s)$ for any $s>1$ ) follows easily from the Euler product representation. Namely,

$$
\zeta_{k}(2)=\prod_{\mathfrak{p}}\left(1-\frac{1}{N \mathfrak{p}^{2}}\right)^{-1}=\prod_{p} \prod_{\mathfrak{p} \mid p}\left(1-\frac{1}{N \mathfrak{p}^{2}}\right)^{-1},
$$


where $\mathfrak{p}$ runs through the set of nonzero prime ideals in $\mathcal{O}_{k}$ and $p$ through all rational primes. Suppose that $\mathfrak{p}_{1}, \ldots, \mathfrak{p}_{t}$ are the prime divisors of $p \mathcal{O}_{k}$ and $N \mathfrak{p}_{i}=p^{f_{i}}$. Then

$$
\prod_{\mathfrak{p} \mid p}\left(1-\frac{1}{N \mathfrak{p}^{2}}\right)^{-1}=\prod_{i=1}^{t}\left(\frac{p^{2 f_{i}}}{p^{2 f_{i}}-1}\right)=\frac{p^{2\left(f_{1}+\cdots+f_{t}\right)}}{\prod_{i=1}^{t}\left(p^{2 f_{i}}-1\right)} .
$$

We have an obvious inequality $\prod_{i=1}^{t}\left(p^{2 f_{i}}-1\right)<p^{2\left(f_{1}+\cdots+f_{t}\right)}-1$. Hence

$$
\prod_{\mathfrak{p} \mid p}\left(1-\frac{1}{N \mathfrak{p}^{2}}\right)^{-1}>\frac{p^{2\left(f_{1}+\cdots+f_{t}\right)}}{p^{2\left(f_{1}+\cdots+f_{t}\right)}-1}=\left(1-\frac{1}{p^{2\left(f_{1}+\cdots+f_{t}\right)}}\right)^{-1} .
$$

Finally, the fundamental identity for prime ideals (see Neukirch [13, Proposition I.(8.2)]) implies that $f_{1}+\cdots+f_{t} \leq m$ and we have $\prod_{\mathfrak{p} \mid p}\left(1-1 /\left(N \mathfrak{p}^{2}\right)\right)^{-1}>\left(1-1 / p^{2 m}\right)^{-1}$ and the stated inequality follows. (Obviously, $\zeta_{\mathbb{Q}}(s)=1+1 / 2^{s}+\cdots>1$ for any real $s>1)$.

Using the above inequalities, (8) implies the relation

$$
2^{n}>2^{n-2 m+1} \pi^{-2 m} d_{k}^{3 / 2}
$$

or equivalently

$$
d_{k}<\left(\frac{(2 \pi)^{2 m}}{2}\right)^{2 / 3}
$$

For any totally real field $K$ of degree $m$ and discriminant $d_{K}$ let $\delta_{K}=d_{K}^{1 / m}$ be its so-called root discriminant and let

$$
\delta_{\min }^{r}(m)=\min \left\{\delta_{K} \mid K \text { is totally real of degree } m\right\} .
$$

The equation (9) implies that for our purposes relevant fields $k$ must satisfy $\delta_{k}<$ $f(m)=(2 \pi)^{4 / 3} / 2^{2 / 3 m}$. The function $f$ is increasing in $m$ but $f(m)<(2 \pi)^{4 / 3}<$ 11.6 for all $m$. A Odlyzko proved lower bounds for $\delta_{\min }^{r}(m)$ and from his work we know that for any $m \geq 9$ the inequality $\delta_{\text {min }}^{r}(m)>11.823$ holds (see Odlyzko [14]). We conclude that $m \leq 8$. But we can improve this knowing the exact value of the minimal root discriminant of a totally real field of degree less than or equal to 8 which has been determined by $\mathbf{J}$ Voight in [24, Table 3]. The values of $\delta_{\min }^{r}(m)$ and $f(m)$ are compared in Table 1.

\begin{tabular}{c|ccccc}
$m$ & 8 & 7 & 6 & 5 & 4 \\
\hline$\delta_{\min }^{r}(m)$ & 11.385 & 11.051 & 8.182 & 6.809 & 5.189 \\
$f(m)$ & 10.943 & 10.853 & 10.734 & 10.570 & 10.329
\end{tabular}

Table 1: Root discriminant bounds

Altogether, we have: 
Lemma 3.4 If $\Gamma_{\mathcal{O}}^{1}$, with $\mathcal{O}$ a maximal order in the quaternion algebra $A\left(k ; m, n, d_{A}\right)$ as above, contains a lattice of a fake $\left(\mathbb{P}^{1}\right)^{n}$, then $n \leq m=[k: \mathbb{Q}] \leq 6$.

Since the dimension $n$ of an arithmetic fake product is always less than or equal to the degree $m$ of the center field $k$ of the defining quaternion algebra, any irreducible fake $\left(\mathbb{P}^{1}\right)^{n}$ whose fundamental group satisfies condition (7) is either 2-, 4- or 6dimensional. As already remarked, the 2-dimensional examples are discussed in [8] (see also references therein) in greater generality. Moreover we can prove:

Theorem 3.5 There are no irreducible fake $\left(\mathbb{P}^{1}\right)^{6} X_{\Gamma}$ such that $\Gamma$ satisfies (7).

Proof Assume that $X_{\Gamma}$ is an irreducible fake $\left(\mathbb{P}^{1}\right)^{6}$ such that $\Gamma<\Gamma_{\mathcal{O}}^{1}$ and $\mathcal{O}^{1}$ is a maximal order in $A=A\left(k ; 6,6, d_{A}\right)$. According to Lemma 3.4 the totally real number field $k$ is of degree 6 with discriminant $d_{k} \leq(10.734)^{6} \approx 1529570.6$. Finally, $\operatorname{vol}\left(X_{\Gamma_{\mathcal{O}}^{1}}\right)=2 \zeta_{k}(-1) \cdot \prod_{\mathfrak{p} \mid d_{A}}(N \mathfrak{p}-1) \leq 2^{6}$. As $\Gamma$ is cocompact, $A$ is a division algebra and therefore there must be at least one finite place of $k$ ramified in $A$ (by assumption, $A$ is unramified at all infinite places of $k$ ). Since the number of ramified places is even (by the reciprocity law for Hilbert-symbols (see Vignéras [23, Corollaire 3.3, page 76])) there must be at least two such finite places. In Table 5 (see Section 5), we collected the needed invariants of all totally real sextic fields with discriminant less than 1529570.6 which were computed with PARI. The list of these fields has been produced by J Voight (see [25]). With the knowledge of all these values, case by case analysis shows that there is no totally indefinite division quaternion algebra $A$ over a sextic real field satisfying $2 \zeta_{k}(-1) \cdot \prod_{\mathfrak{p} \mid d_{A}}(N \mathfrak{p}-1) \leq 2^{6}$. We note that the last condition bounds the value of $\prod_{\mathfrak{p} \mid d_{A}}(N \mathfrak{p}-1)=\prod_{\mathfrak{p} \mid d_{A}}\left(p^{f(\mathfrak{p} / p)}-1\right)$, with $f(\mathfrak{p} / p)$ the inertia degree of $\mathfrak{p}$, and thus restricts the set of possible prime ideals $\mathfrak{p}$ at which $A$ ramifies.

We will now focus on 4-dimensional fake products.

Theorem 3.6 Let $A=A\left(k ; m, 4, d_{A}\right)$ be a quaternion algebra over a totally real number field $k$ unramified at 4 archimedean places of $k$ and $\mathcal{O}$ a maximal order in $A$. Assume that $\Gamma<\Gamma_{\mathcal{O}}^{1}$ is a finite-index torsion-free subgroup such that $X_{\Gamma}=\Gamma \backslash \mathbb{H}^{4}$ is a fake product of projective lines. Then the pair $\left(k, d_{A}\right)$ (determining the isomorphism class of $A$ uniquely) belongs to the list in Table 2.

There, the reduced discriminant $d_{A}=\prod \mathfrak{p}_{p}$ is a (possibly empty) formal product of suitably chosen prime ideals $\mathfrak{p}_{p}$ lying over a rational prime $p$.

Remark 3.7 To specify what "suitably chosen" in the above Theorem 3.6 means, let us consider an example. Let $k$ be the totally real field of degree 4 and discriminant 


\begin{tabular}{cccc}
$d_{k}$ & defining polynomial & $\zeta_{k}(-1)$ & $d_{A}$ \\
\hline 1957 & $x^{4}-4 x^{2}-x+1$ & $2 / 3$ & $\mathfrak{p}_{3} \mathfrak{p}_{7}$ \\
2000 & $x^{4}-5 x^{2}+5$ & $2 / 3$ & $\mathfrak{p}_{2} \mathfrak{p}_{5}$ \\
2304 & $x^{4}-4 x^{2}+1$ & 1 & $\mathfrak{p}_{2} \mathfrak{p}_{3}$ \\
38569 & $x^{5}-5 x^{3}+4 x-1$ & $-8 / 3$ & $\mathfrak{p}_{7}$ \\
106069 & $x^{5}-2 x^{4}-4 x^{3}+7 x^{2}+3 x-4$ & -16 & $\mathfrak{p}_{2}$ \\
453789 & $x^{6}-x^{5}-6 x^{4}+6 x^{3}+8 x^{2}-8 x+1$ & $16 / 3$ & $\varnothing$ \\
1387029 & $x^{6}-3 x^{5}-2 x^{4}+9 x^{3}-x^{2}-4 x+1$ & 32 & $\varnothing$ \\
1397493 & $x^{6}-3 x^{5}-3 x^{4}+10 x^{3}+3 x^{2}-6 x+1$ & 32 & $\varnothing$
\end{tabular}

Table 2

$d_{k}=38569$. Then there are two prime ideals of $k$ lying over 7 , but one with inertia degree 1 and the other with inertia degree 4 (see Table 4, Section 5). Only the first prime ideal can be taken as the prime where $A$, the quaternion algebra defining a fake $\left(\mathbb{P}^{1}\right)^{4}$, ramifies, but not the latter.

Proof of Theorem 3.6 Let $A$ and $\Gamma<\Gamma_{\mathcal{O}}^{1}$ as above be given. Then by Lemma 3.4, $m=[k: \mathbb{Q}] \leq 6$ and the root discriminant $\delta_{k}$ satisfies $\delta_{k} \leq f(m)$ with the value $f(m)$ from Table 1 . We know that $e\left(X_{\Gamma}\right)=16=\left[\Gamma_{\mathcal{O}}^{1}: \Gamma\right] e\left(X_{\Gamma_{\mathcal{O}}^{1}}\right)=2^{5-m+1} \zeta_{k}(-1) \cdot$ integer. Hence, $k$ satisfies the condition

$$
\frac{16}{2^{5-m} \zeta_{k}(-1)} \in \mathbb{N}
$$

In the already mentioned Table 5 from Section 5 , we find all the sextic fields $k$ satisfying $\delta_{k} \leq f(6)$. Additionally, Tables 4 and 3 (see Section 5) contain all the totally real quintic and quartic fields with $\delta_{k} \leq f(m)$ and satisfying the condition (10). Next, note that for each finite place $\mathfrak{p}$ of $k$ which divides the reduced discriminant $d_{A}$, the value $N \mathfrak{p}-1=p^{f(\mathfrak{p} / p)}-1$ divides $16 /\left(2^{5-m} \zeta_{k}(-1)\right)$. The relevant values for $f(\mathfrak{p} / p)$ are given in Tables 5, 4 and 3. Finally, for $m=4,5$, the quaternion algebra (which is assumed to be a skew field) has to be ramified at least at one finite place of $k$ and if $m=4, A$ ramifies at least at two finite places.

\section{Examples}

Let $\xi=\xi_{20}$ be a primitive $20^{\text {th }}$ root of unity, $K=\mathbb{Q}(\xi)$ the corresponding cyclotomic field and $k=\mathbb{Q}\left(\xi+\xi^{-1}\right)=\mathbb{Q}(\cos (\pi / 10))$ the maximal totally real subfield of $K$. The field $k$ is defined by the polynomial $P(x)=x^{4}-5 x^{2}+5$. We summarize some relevant facts about $k$. 
Lemma 4.1 The field $k$ is a totally real abelian quartic field of discriminant $d_{k}=2000$. The small rational primes have the following prime ideal decomposition in $k$ :

- $2 \mathcal{O}_{k}=\mathfrak{p}_{2}^{2}$, where $\mathfrak{p}_{2}$ is a prime ideal in $\mathcal{O}_{k}$ with norm $N \mathfrak{p}_{2}=4$.

- $3 \mathcal{O}_{k}=\mathfrak{p}_{3}$ is inert.

- $5 \mathcal{O}_{k}=\mathfrak{p}_{5}^{4}$ is totally ramified in $k$.

The value of Dedekind zeta function $\zeta_{k}(s)$ at $s=-1$ is $\zeta_{k}(-1)=\frac{2}{3}$.

Proof Either one consults the Table 3 in Section 5 or one applies directly some of the well-known facts about cyclotomic fields. Let us briefly explain the second approach: Let $\xi_{n}$ be a primitive $n^{\text {th }}$ root of unity. Then, the extension $\mathbb{Q}\left(\xi_{n}\right) / \mathbb{Q}\left(\xi_{n}+\xi_{n}^{-1}\right)$ is unramified at all the finite places of $k$ for $n$ not a prime power (see Washington [26, Proposition 2.15]). Also, a rational prime $p$ factorizes in $\mathbb{Q}\left(\xi_{n}\right)$ as

$$
p \mathcal{O}_{\mathbb{Q}\left(\xi_{n}\right)}=\left(\mathfrak{Q}_{1} \cdots \mathfrak{Q}_{r}\right)^{\varphi\left(p^{v p(n)}\right)},
$$

where all $\mathfrak{Q}_{i}$ are of residual degree $f_{p}=\min \left\{f \mid p^{f} \equiv 1 \bmod n / p^{v_{p}(n)}\right\}$ and $v_{p}(\cdot)$ is the normalized $p$-valuation [13, Proposition I(10.3)]. Recall again the fundamental identity for prime ideals [13, Proposition I.(8.2)] which in this case states that $f_{p} \varphi\left(p^{v_{p}(n)}\right) r=\varphi(n)$. It follows for $\xi=\xi_{20}$ that the principal ideal $2 \mathcal{O}_{\mathbb{Q}(\xi)}=\mathfrak{P}_{2}^{2}$ is a square of a prime ideal in $\mathcal{O}_{\mathbb{Q}(\xi)}$. As $\mathbb{Q}(\xi) / k$ is unramified, the stated decomposition of 2 in $\mathcal{O}_{k}$ follows. The above shows also that 3 is inert in $\mathcal{O}_{\mathbb{Q}(\xi)}$ hence inert in $\mathcal{O}_{k}$. Finally, we find that $5 \mathcal{O}_{\mathbb{Q}(\xi)}=\left(\mathfrak{P P}^{\prime}\right)^{4}$ is a product of two prime powers. Again, as the extension $\mathbb{Q}(\xi) / k$ is unramified, the fundamental identity allows only the stated possibility for the decomposition of 5 in $\mathcal{O}_{k}$. For the computation of the zeta value, one can use the formula for $\zeta_{k}(-1)$ as the product of values $L(-1, \chi)$, where $\chi$ runs over all even Dirichlet characters modulo 20 (a Dirichlet character $\chi$ is even if $\chi(-1)=1$ ) and the formula $L(-1, \chi)=-B_{2, \chi} / 2$ in terms of the generalized Bernoulli number $B_{2, \chi}$ associated with $\chi$ [26, Theorems 4.2 and 4.3]. The general formula for the values $B_{2, \chi}$ can be found for instance in [26, Proposition 4.1]. If $\chi$ is a nontrivial even character with conductor $N$ there is a simple expression for $B_{2, \chi}$, namely $B_{2, \chi}=\frac{1}{N} \sum_{a=1}^{N} \chi(a) a^{2}$ (see for instance [26, Exercise 4.2]). Now, there are four even Dirichlet characters mod 20: the trivial character $\chi_{0}$ with $B_{2, \chi_{0}}=1 / 6$, a real character $\chi_{1}$ with conductor 5 given by $\chi_{1}(11)=1, \chi_{1}(17)=-1$ contributing $B_{2, \chi_{1}}=4 / 5$ and two conjugate even characters $\chi_{2}, \bar{\chi}_{2}$ with conductor 20 defined by $\chi_{2}(11)=-1, \chi_{2}(17)=\sqrt{-1}$ contributing $B_{2, \chi_{2}}=4 \sqrt{-1}+8$. Altogether we get $\zeta_{k}(-1)=\frac{1}{12} \frac{2}{5}(2 \sqrt{-1}+4)(-2 \sqrt{-1}+4)=\frac{2}{3}$.

Now let $A=A\left(k ; 4,4, \mathfrak{p}_{2}, \mathfrak{p}_{5}\right)$ be the quaternion algebra over $k$, ramified exactly at the two places $\mathfrak{p}_{2}$ and $\mathfrak{p}_{5}$. 
Theorem 4.2 Let $\mathcal{O} \subset A\left(k ; 4,4, \mathfrak{p}_{2}, \mathfrak{p}_{5}\right)$ be a maximal order. Then, $X_{\Gamma_{\mathcal{O}}^{1}}$ is a fourdimensional fake product of projective lines.

Proof By Lemma 2.1, $e\left(X_{\Gamma_{\mathcal{O}}^{1}}\right)=2 \zeta_{k}(-1)\left(N \mathfrak{p}_{2}-1\right)\left(N \mathfrak{p}_{5}-1\right)$, and Lemma 4.1 gives immediately the asked value $e\left(X_{\Gamma_{\mathcal{O}}}^{1}\right)=2 \cdot \frac{2}{3}\left(2^{2}-1\right)(5-1)=16=2^{4}$. We need to prove that $X_{\Gamma_{\mathcal{O}}^{1}}$ is smooth. For this we will exclude the existence of elements of finite order in $\Gamma_{\mathcal{O}}^{1}$. Note that there exists a torsion element in $\Gamma_{\mathcal{O}}^{1}$ if and only if there is an embedding of $\mathcal{O}_{k}[\rho]$ into $\mathcal{O}$, where $\rho$ is some primitive root of unity. In particular, every torsion corresponds to a (commutative) subfield $k(\xi) \subset A$. In order to show the claim we will exclude the possibility of an embedding $k(\rho) \hookrightarrow A$. It is sufficient to consider only primitive $p^{\text {th }}$ roots of unity $\rho$, where $p$ is an odd prime or $p=4$. Since $[k: \mathbb{Q}]=4$, and $k(\rho) / k$ is at most a quadratic extension, $k(\rho) \subset A$ is possible at most for $p=3,4$ or 5. By the classical embedding theorem of Hasse [23, Theoreme 3.8, page 78], $k(\rho)$ can be embedded in $A$ if and only if each prime $\mathfrak{p} \mid d_{A}$ is nonsplit in $k(\rho)$. Let us consider $\rho=\xi_{3}$ and show that $\mathfrak{p}_{2} \subset \mathcal{O}_{k}$, the prime ideal over 2, splits in the field $L=k\left(\xi_{3}\right)$. The field $L=k(\sqrt{-3})$ is a subfield of $\mathbb{Q}\left(\xi_{60}\right)$, and by the facts from the general theory of cyclotomic fields used in the proof of Lemma $4.1,2 \mathcal{O}_{\mathbb{Q}\left(\xi_{60}\right)}=\mathfrak{Q}_{1}^{2} \mathfrak{Q}_{2}^{2}$, where $\mathfrak{Q}_{1,2}$ are both of inertia degree 4 over 2 . The primes $\mathfrak{Q}_{1}$ and $\mathfrak{Q}_{2}$ are Galois conjugate by the automorphism $\sqrt{-3} \mapsto-\sqrt{-3}$, since $2 \mathcal{O}_{\mathbb{Q}\left(\xi_{20}\right)}=\mathfrak{q}^{2}$ with inertia degree $f(\mathfrak{q} / 2)=4$. Lemma 4.1 implies that $\mathfrak{p}_{2}$ is split in $k\left(\xi_{3}\right)$ and therefore $k\left(\xi_{3}\right)$ is not a subfield of $A$. Consider next $L=k(\rho)$ with $\rho=\xi_{4}$ or $\rho=\xi_{5}$. Then, $L=K=k\left(\xi_{20}\right)$, and we can use same kind of arguments: namely, we know that $K / k$ is unramified at all the finite places and $5 \mathcal{O}_{K}=\mathfrak{P}^{4} \cdot \mathfrak{P}^{\prime 4}$ with two prime ideals $\mathfrak{P}, \mathfrak{P}^{\prime}$ in $K$ with $f(\mathfrak{P} / 5)=f\left(\mathfrak{P}^{\prime} / 5\right)=1$. By Lemma $4.1,5 \mathcal{O}_{k}=\mathfrak{p}_{5}^{4}$, hence $\mathfrak{p}_{5}=\mathfrak{P P}^{\prime}$ is split in $K$ and $K$ is not a subfield of $A$.

Let us present another example. Let $\xi_{24}$ be a primitive $24^{\text {th }}$ root of unity, $L=\mathbb{Q}\left(\xi_{24}\right)$ the corresponding cyclotomic field and $\ell=\mathbb{Q}\left(\xi_{24}+\xi_{24}^{-1}\right)$ the maximal totally real subfield of $L$. Then, as in the example before we have the following elementary result, which can be proved in the same way as Lemma 4.1.

Lemma 4.3 We have that $\ell$ is a quartic field of discriminant $d_{\ell}=2304$ and $\zeta_{\ell}(-1)=1$. We have the following prime ideal decomposition of rational primes in $\mathcal{O}_{\ell}$ :

- $2 \mathcal{O}_{\ell}=\mathfrak{p}_{2}^{4}$.

- $3 \mathcal{O}_{\ell}=\mathfrak{p}_{3}^{2}$.

- $5 \mathcal{O}_{\ell}=\mathfrak{p}_{5} \mathfrak{p}_{5}^{\prime}$.

Theorem 4.4 Let $A=A\left(\ell ; 4, \mathfrak{p}_{2}, \mathfrak{p}_{3}\right)$ be the totally indefinite quaternion algebra over $\ell$ of reduced discriminant $d_{A}=\mathfrak{p}_{2} \mathfrak{p}_{3}$ and $\mathcal{O}$ a maximal order in $A$. Then $X_{\Gamma_{\mathcal{O}}^{1}}$ is an irreducible four-dimensional fake product of projective lines. 
Proof The proof goes along the lines of the proof of Theorem 4.2. The equality $e\left(X_{\Gamma_{\mathcal{O}}^{1}}\right)=2(2-1)\left(3^{2}-1\right)=16$ follows immediately from Lemmas 4.3 and 2.1. For proving the smoothness of $X_{\Gamma_{\mathcal{O}}^{1}}$, note that $\ell\left(\xi_{3}\right)$ and $\ell\left(\xi_{4}\right)$ coincide with the cyclotomic field $\mathbb{Q}\left(\xi_{24}\right)$ where $\mathfrak{p}_{3} \subset \mathcal{O}_{\ell}$, the prime ideal over 3 is split. This shows that $\ell\left(\xi_{3}\right)$ and $\ell\left(\xi_{4}\right)$ cannot be contained in $A$. Thus $X_{\Gamma_{\mathcal{O}}^{1}}$ is smooth.

After giving the examples, let us show that all the other virtual candidates from Theorem 3.6 do not give rise to a lattice of a fake product.

Theorem 4.5 Besides the two examples above, no other quaternion algebra $A$ contains a lattice of a fake $\left(\mathbb{P}^{1}\right)^{n}$ which is a finite-index subgroup of the norm-one group of a maximal order in $A$.

Proof We will exclude the only possible quaternion algebras from Theorem 3.6 by showing the corresponding lattices are never torsion free (if they exist). Consider for instance $A\left(k_{1957}, 4,4, \mathfrak{p}_{3} \mathfrak{p}_{7}\right)$ with $k_{1957}$, the totally real quartic field with discriminant 1957, which has the smallest discriminant among the possible fields. From Table $3, \operatorname{vol}\left(\Gamma_{\mathcal{O}}^{1}\right)=\frac{4}{3} \cdot(3-1)(7-1)=16$, hence $\Gamma_{\mathcal{O}}^{1}$ is the only candidate for a lattice of a 4-dimensional fake product. But we can show $\Gamma_{\mathcal{O}}^{1}$ contains an element of order 2 which comes from an embedding of $\xi_{4}$ into $\mathcal{O}$. Namely, let $K=k_{1957}\left(\xi_{4}\right)$; then $K$ is a totally complex field of discriminant $980441344=2^{8} 19^{2} 103^{2}$ defined by $x^{8}-4 x^{6}+2 x^{5}+16 x^{4}-12 x^{3}+25 x^{2}-12 x+37$ (via PARI). Considering the factorization of this polynomial modulo 3, the rational prime 3 factorizes in $K$ as $\mathfrak{q}_{3} \mathfrak{q}_{3}^{\prime}$ as a product of two prime ideals. Since 3 is also a product $\mathfrak{p}_{3} \mathfrak{p}_{3}^{\prime}$ of two prime ideals in $k$ (compare Table 3 ), $\mathfrak{p}_{3}$ remains prime in $K$ and hence by the embedding theorem [23, Theoreme 3.8, page 78] $\xi_{4}$ can be embedded into $A$. But this embedding can even be chosen in such a way that $\xi_{4}$ lies in $\mathcal{O}$ (see Shimura [20, Proposition 2.8]) so $\xi_{4}$ gives rise to a torsion in $\Gamma_{\mathcal{O}}^{1}$. In all the other cases, except $A=A\left(k_{453789}, 6,4, \varnothing\right)$, the same kind of arguments work: the only possible lattice is $\Gamma_{\mathcal{O}}^{1}$ itself, but which is never torsion free. In the case $A=A\left(k_{453789}, 6,4, \varnothing\right)$, where $k_{453789}$ is the totally real sextic field of discriminant 453789 , the possible lattice of a 4-dimensional fake product is a subgroup of index 6 in $\Gamma_{\mathcal{O}}^{1}$. Now recall the following elementary group-theoretic result (see Brown [5, IX 9.2] for a proof of a more precise result): If $H, G^{\prime}<G$ are groups with $H$ finite and $G^{\prime}$ torsion free, then the index $\left[G: G^{\prime}\right]$ is divisible by $|H|$. On the other hand, $k_{453789}=\mathbb{Q}\left(\xi_{21}+\xi_{21}^{-1}\right)$ is the maximal totally real number field of the cyclotomic field $\mathbb{Q}\left(\xi_{21}\right)$. Since $\mathbb{Q}\left(\xi_{21}\right)$ is a totally imaginary quadratic extension of $k_{453789}$ and $A$ is unramified at all the finite places, $\xi_{21}$ can be embedded into $\mathcal{O}$ and the index of a torsion-free subgroup in $\Gamma_{\mathcal{O}}^{1}$ must be divisible by 21 which contradicts the assumption $\left[\Gamma_{\mathcal{O}}^{1}: \Gamma\right]=6$. 
Remark 4.6 More precisely we can say that up to isomorphism, the two examples of fake $\left(\mathbb{P}^{1}\right)^{4}$ are the only examples of irreducible fake $\left(\mathbb{P}^{1}\right)^{n}$ with $n \geq 4$ whose fundamental group is contained in $\Gamma_{\mathcal{O}}^{1}$. This follows from the fact that there is a single conjugacy class of maximal order inside $A\left(\mathbb{Q}\left(\xi_{20}+\xi_{20}^{-1}\right), 4,4, \mathfrak{p}_{2} \mathfrak{p}_{5}\right)$ and $A\left(\mathbb{Q}\left(\xi_{24}+\right.\right.$ $\left.\left.\xi_{24}^{-1}\right), 4,4, \mathfrak{p}_{2} \mathfrak{p}_{3}\right)$ since both fields have class number one (see [11, Section 6.7, (6.13)]).

\section{Tables}

In this section we list the tables of invariants of quaternion algebras related to lattices of fake products of projective lines used in previous sections.

\begin{tabular}{ccccccccc}
$d_{k}$ & defining polynomial & $\zeta_{k}(-1)$ & $f\left(\frac{\mathfrak{p}}{2}\right)$ & $f\left(\frac{\mathfrak{p}}{3}\right)$ & $f\left(\frac{\mathfrak{p}}{5}\right)$ & $f\left(\frac{\mathfrak{p}}{7}\right)$ & $f\left(\frac{\mathfrak{p}}{11}\right)$ & $f\left(\frac{\mathfrak{p}}{13}\right)$ \\
\hline 725 & $x^{4}-x^{3}-3 x^{2}+x+1$ & $\frac{2}{15}$ & 4 & 4 & 2 & $2 ; 2$ & $1 ; 1 ; 2$ & $2 ; 2$ \\
1125 & $x^{4}-x^{3}-4 x^{2}+4 x+1$ & $\frac{4}{15}$ & 4 & 2 & & 4 & $2 ; 2$ & \\
1957 & $x^{4}-4 x^{2}-x+1$ & $\frac{2}{3}$ & 4 & $1 ; 3$ & 4 & $1 ; 3$ & & \\
2000 & $x^{4}-5 x^{2}+5$ & $\frac{2}{3}$ & 2 & 4 & 1 & 4 & & 4 \\
2225 & $x^{4}-x^{3}-5 x^{2}+2 x+4$ & $\frac{4}{5}$ & $2 ; 2$ & 4 & & & $2 ; 2$ & \\
2304 & $x^{4}-4 x^{2}+1$ & 1 & 1 & 2 & 22 & & & \\
2525 & $x^{4}-2 x^{3}-4 x^{2}+5 x+5$ & $\frac{4}{3}$ & 4 & 4 & $1 ; 1$ & 4 & & \\
2624 & $x^{4}-2 x^{3}-3 x^{2}+2 x+1$ & 1 & 2 & 4 & & $1 ; 1 ; 2$ & & \\
2777 & $x^{4}-x^{3}-4 x^{2}+x+2$ & $\frac{4}{3}$ & $1 ; 3$ & 4 & & 4 & & \\
3600 & $x^{4}-2 x^{3}-7 x^{2}+8 x+1$ & $\frac{8}{5}$ & & & & & & \\
3981 & $x^{4}-x^{3}-4 x^{2}+2 x+1$ & 2 & 4 & $1 ; 2$ & $1 ; 3$ & & & \\
4205 & $x^{4}-x^{3}-5 x^{2}-x+1$ & 2 & 4 & 4 & $1 ; 2$ & & & \\
4352 & $x^{4}-6 x^{2}-4 x+2$ & $\frac{8}{3}$ & 1 & 4 & & & & \\
9909 & $x^{4}-6 x^{2}-3 x+3$ & 8 & 4 & & & & & \\
10512 & $x^{4}-7 x^{2}-6 x+1$ & 8 & 2 & & & & &
\end{tabular}

Table 3: Totally real quartic fields with root discriminant less than or equal to 10.3 and with the property that $16 / 2 \zeta_{k}(-1)$ is an integer: each entry in the column $f(\mathfrak{p} / p)$ is the inertia degree of a prime ideal $\mathfrak{p}$ dividing $p \mathcal{O}_{k}$; the number of entries is the number of prime ideals dividing $p \mathcal{O}_{k}$. 
The invariants are obtained as follows. First we collect all defining polynomials of number fields of degree $m \leq 6$ with root discriminant less than $f(m)$ which can be found in Cohen [2] and Voight [25]. The PARI procedure nfinit->idealprimedec gives the decomposition of small rational primes in $k$. We use the PARI procedure zetakinit->zetak to compute the values $\zeta_{k}(-1)$. More precisely, PARI a priori computes an approximation of the true value of $\zeta_{k}(2)$ by computing the truncated Euler product

$$
\prod_{p \leq x} \prod_{\mathfrak{p} \mid p}\left(1-N \mathfrak{p}^{-2}\right)^{-1}
$$

The accuracy depends on the choice of $x$ but the precision of 5-10 decimal digits is certain and this turns out to be enough in given cases. We can namely control the result knowing some properties of the zeta values $\zeta_{k}(-1)$. Recall that by the theorem of Klingen and Siegel [21], $\zeta_{k}(-1)$ is a rational number and moreover we have information on primes dividing the denominator of $\zeta_{k}(-1)$ and its size. By [21, page 89] we know that the denominator of $2^{m} \zeta_{k}(-1)$ divides a certain integer $c_{2 m}$ which in given cases is product of small primes (if $m=4$ then $p=2,3,5$ ).

Hence we have an upper bound $B$ such that $B \zeta_{k}(-1)$ is an integer. The functional equation implies that $B \zeta_{k}(-1)=B \zeta_{k}(2) \cdot(-1)^{m} 2^{-m} \pi^{-2 m} d_{k}^{3 / 2}$ is an integer. So with an approximation $Z_{k}(2)$ we would take the closest integer to $B Z_{k}(2) \cdot(-1)^{m} 2^{-m} \pi^{-2 m} d_{k}^{3 / 2}$ to obtain the true value. Consider for instance the quartic field $k$ with discriminant $d_{k}=725$. PARI gives us the value $Z_{k}(2)=1.0369329880 \ldots$ By Siegel's theorem we know that only $2,3,5$ can divide the denominator of $\zeta_{k}(-1)$. Hence from the value

$$
30 Z_{k}(2) 2^{-4} \pi^{-8} 725^{3 / 2}=3.9999999997
$$

we find that $\zeta_{k}(-1)=\frac{4}{30}=\frac{2}{15}$ is the correct value.

\begin{tabular}{ccccccccc}
$d_{k}$ & defining polynomial & \multicolumn{1}{c}{$\zeta_{k}(-1)$} & $f\left(\frac{\mathfrak{p}}{2}\right)$ & $f\left(\frac{\mathfrak{p}}{3}\right)$ & $f\left(\frac{\mathfrak{p}}{5}\right)$ & $f\left(\frac{\mathfrak{p}}{7}\right)$ & $f\left(\frac{\mathfrak{p}}{11}\right)$ & $f\left(\frac{\mathfrak{p}}{13}\right)$ \\
\hline 24217 & $x^{5}-5 x^{3}-x^{2}+3 x+1$ & $-\frac{4}{3}$ & 5 & 5 & $1 ; 4$ & 5 & $2 ; 3$ & $2 ; 3$ \\
36497 & $x^{5}-2 x^{4}-3 x^{3}+5 x^{2}+x-1$ & $-\frac{8}{3}$ & 5 & 5 & $2 ; 3$ & $2 ; 3$ & & \\
38569 & $x^{5}-5 x^{3}+4 x-1$ & $-\frac{8}{3}$ & 5 & 5 & 5 & $1 ; 4$ & & \\
81509 & $x^{5}-x^{4}-5 x^{3}+3 x^{2}+5 x-2$ & $-\frac{32}{3}$ & & & & & &
\end{tabular}

Table 4: Totally real quintic fields with root discriminant less than or equal to 10.570 and with the property that $16 / \zeta_{k}(-1)$ is an integer: each entry in the column $f(\mathfrak{p} / p)$ is the inertia degree of a prime ideal $\mathfrak{p}$ dividing $p \mathcal{O}_{k}$; the number of entries is the number of prime ideals dividing $p \mathcal{O}_{k}$. 


\begin{tabular}{|c|c|c|c|c|c|c|c|}
\hline$d_{k}$ & defining polynomial & $\zeta_{k}(-1)$ & $f\left(\frac{\mathfrak{p}}{2}\right)$ & $f\left(\frac{\mathfrak{p}}{3}\right)$ & $f\left(\frac{\mathfrak{p}}{5}\right)$ & $f\left(\frac{\mathfrak{p}}{7}\right)$ & $f\left(\frac{\mathfrak{p}}{11}\right)$ \\
\hline 300125 & $x^{6}-x^{5}-7 x^{4}+2 x^{3}+7 x^{2}-2 x-1$ & $\frac{296}{105}$ & 6 & 6 & 3 & 2 & $3 ; 3$ \\
\hline 371293 & $x^{6}-x^{5}-5 x^{4}+4 x^{3}+6 x^{2}-3 x-1$ & $\frac{152}{39}$ & 6 & $3 ; 3$ & $2 ; 2 ; 2$ & 6 & \\
\hline 434581 & $x^{6}-2 x^{5}-4 x^{4}+5 x^{3}+4 x^{2}-2 x-1$ & $\frac{104}{21}$ & 6 & $3 ; 3$ & $3 ; 3$ & 2 & \\
\hline 453789 & $x^{6}-x^{5}-6 x^{4}+6 x^{3}+8 x^{2}-8 x+1$ & $\frac{16}{3}$ & 6 & 3 & $3 ; 3$ & 1 & \\
\hline 485125 & $x^{6}-2 x^{5}-4 x^{4}+8 x^{3}+2 x^{2}-5 x+1$ & $\frac{88}{15}$ & 6 & $2 ; 4$ & 3 & & \\
\hline 592661 & $x^{6}-x^{5}-5 x^{4}+4 x^{3}+5 x^{2}-2 x-1$ & 8 & 6 & 6 & $2 ; 4$ & & \\
\hline 703493 & $x^{6}-2 x^{5}-5 x^{4}+11 x^{3}+2 x^{2}-9 x+1$ & $\frac{72}{7}$ & 6 & 6 & & & \\
\hline 722000 & $x^{6}-x^{5}-6 x^{4}+7 x^{3}+4 x^{2}-5 x+1$ & $\frac{56}{5}$ & 2 & 6 & & & \\
\hline 810448 & $x^{6}-3 x^{5}-2 x^{4}+9 x^{3}-5 x+1$ & $\frac{40}{3}$ & 2 & $3 ; 3$ & & & \\
\hline 820125 & $x^{6}-9 x^{4}-4 x^{3}+9 x^{2}+3 x-1$ & $\frac{584}{45}$ & 6 & 2 & & & \\
\hline 905177 & $x^{6}-x^{5}-7 x^{4}+9 x^{3}+7 x^{2}-9 x-1$ & $\frac{320}{21}$ & $3 ; 3$ & 6 & & & \\
\hline 966125 & $x^{6}-x^{5}-6 x^{4}+4 x^{3}+8 x^{2}-1$ & $\frac{256}{15}$ & 6 & & & & \\
\hline 980125 & $x^{6}-x^{5}-6 x^{4}+6 x^{3}+7 x^{2}-5 x-1$ & $\frac{256}{15}$ & 6 & & & & \\
\hline 1075648 & $x^{6}-7 x^{4}+14 x^{2}-7$ & $\frac{416}{21}$ & 3 & & & & \\
\hline 1081856 & $x^{6}-6 x^{4}-2 x^{3}+7 x^{2}+2 x-1$ & 20 & 3 & & & & \\
\hline 1134389 & $x^{6}-2 x^{5}-4 x^{4}+6 x^{3}+4 x^{2}-3 x-1$ & $\frac{64}{3}$ & 6 & & & & \\
\hline 1202933 & $x^{6}-6 x^{4}-2 x^{3}+6 x^{2}+x-1$ & 24 & 6 & & & & \\
\hline 1229312 & $x^{6}-10 x^{4}+24 x^{2}-8$ & $\frac{172}{7}$ & 3 & & & & \\
\hline 1241125 & $x^{6}-7 x^{4}-2 x^{3}+11 x^{2}+7 x+1$ & $\frac{376}{15}$ & 6 & & & & \\
\hline 1259712 & $x^{6}-6 x^{4}+9 x^{2}-3$ & $\frac{248}{9}$ & 3 & & & & \\
\hline 1279733 & $x^{6}-2 x^{5}-6 x^{4}+10 x^{3}+10 x^{2}-11 x-1$ & $\frac{544}{21}$ & 6 & & & & \\
\hline 1292517 & $x^{6}-6 x^{4}-x^{3}+6 x^{2}-1$ & $\frac{232}{9}$ & 6 & & & & \\
\hline 1312625 & $x^{6}-x^{5}-7 x^{4}+7 x^{3}+12 x^{2}-12 x-1$ & $\frac{416}{15}$ & $2 ; 4$ & & & & \\
\hline 1387029 & $x^{6}-3 x^{5}-2 x^{4}+9 x^{3}-x^{2}-4 x+1$ & 32 & 6 & & & & \\
\hline 1397493 & $x^{6}-3 x^{5}-3 x^{4}+10 x^{3}+3 x^{2}-6 x+1$ & 32 & 6 & & & & \\
\hline 1416125 & $x^{6}-2 x^{5}-5 x^{4}+9 x^{3}+6 x^{2}-9 x+1$ & $\frac{152}{5}$ & 2 & & & & \\
\hline 1528713 & $x^{6}-3 x^{5}-3 x^{4}+7 x^{3}+3 x^{2}-3 x-1$ & $\frac{304}{9}$ & $3 ; 3$ & & & & \\
\hline
\end{tabular}

Table 5: Totally real sextic fields with root discriminant less than or equal to 10.734 ; the entries should be read as follows: each entry in the column $f(\mathfrak{p} / p)$ is the inertia degree of a prime ideal $\mathfrak{p}$ dividing $p \mathcal{O}_{k}$; the number of entries is the number of prime ideals dividing $p \mathcal{O}_{k}$. 


\section{References}

[1] I C Bauer, F Catanese, F Grunewald, The classification of surfaces with $p_{g}=q=0$ isogenous to a product of curves, Pure Appl. Math. Q. 4 (2008) 547-586 MR2400886

[2] K Belabas, J Buchmann, H Cohen, F Diaz y Diaz, D Ford, P Létard, M Olivier, M Pohst, A Schwarz, Tables of number fields Available at http:// pari.math.u-bordeaux1.fr/pub/pari/packages/nftables/

[3] M Belolipetsky, B Linowitz, On fields of definition of arithmetic Kleinian reflection groups, II, Int. Math. Res. Not. 2014 (2014) 2559-2571 MR3207375

[4] A Borel, Commensurability classes and volumes of hyperbolic 3-manifolds, Ann. Scuola Norm. Sup. Pisa Cl. Sci. 8 (1981) 1-33 MR616899

[5] K S Brown, Cohomology of groups, Graduate Texts in Math. 87, Springer, New York (1982) MR672956

[6] F Catanese, Fibred surfaces, varieties isogenous to a product and related moduli spaces, Amer. J. Math. 122 (2000) 1-44 MR1737256

[7] T Chinburg, E Friedman, The smallest arithmetic hyperbolic three-orbifold, Invent. Math. 86 (1986) 507-527 MR860679

[8] A Džambić, Fake quadrics from irreducible lattices acting on the product of upper half planes, Math. Ann. 360 (2014) 23-51 MR3263157

[9] F Hirzebruch, Automorphe Formen und der Satz von Riemann-Roch, from: "Symposium internacional de topología algebraica International symposium on algebraic topology", Universidad Nacional Autónoma de México and UNESCO, Mexico City (1958) 129-144 MR0103280

[10] F Hirzebruch, Gesammelte Abhandlungen, Vol. I, Springer, Berlin (1987) MR931775

[11] C Maclachlan, A W Reid, The arithmetic of hyperbolic 3-manifolds, Graduate Texts in Math. 219, Springer, New York (2003) MR1937957

[12] Y Matsushima, G Shimura, On the cohomology groups attached to certain vector valued differential forms on the product of the upper half planes, Ann. of Math. 78 (1963) 417-449 MR0155340

[13] J Neukirch, Algebraic number theory, Grundl. Math. Wissen. 322, Springer, Berlin (1999) MR1697859

[14] A Odlyzko, Discriminant bounds, tables of calculations (1976) Available at http:// www.dtc.umn.edu/ odlyzko/unpublished/index.html

[15] G Prasad, S-K Yeung, Fake projective planes, Invent. Math. 168 (2007) 321-370 MR2289867

[16] G Prasad, S-K Yeung, Arithmetic fake projective spaces and arithmetic fake Grassmannians, Amer. J. Math. 131 (2009) 379-407 MR2503987 
[17] G Prasad, S-K Yeung, Addendum to "Fake projective planes" Invent. Math. 168 (2007) 321-370, Invent. Math. 182 (2010) 213-227 MR2672284

[18] G Prasad, S-K Yeung, Nonexistence of arithmetic fake compact Hermitian symmetric spaces of type other than $A_{n}(n \leq 4)$, J. Math. Soc. Japan 64 (2012) 683-731 MR2965425

[19] I H Shavel, A class of algebraic surfaces of general type constructed from quaternion algebras, Pacific J. Math. 76 (1978) 221-245 MR0572981

[20] G Shimura, Construction of class fields and zeta functions of algebraic curves, Ann. of Math. 85 (1967) 58-159 MR0204426

[21] C L Siegel, Berechnung von Zetafunktionen an ganzzahligen Stellen, Nachr. Akad. Wiss. Göttingen Math.-Phys. Kl. II 1969 (1969) 87-102 MR0252349

[22] M-F Vignéras, Invariants numériques des groupes de Hilbert, Math. Ann. 224 (1976) 189-215 MR0429755

[23] M-F Vignéras, Arithmétique des algèbres de quaternions, Lecture Notes in Math. 800, Springer, Berlin (1980) MR580949

[24] J Voight, Enumeration of totally real number fields of bounded root discriminant, from: "Algorithmic number theory", (A J van der Poorten, A Stein, editors), Lecture Notes in Comput. Sci. 5011, Springer, Berlin (2008) 268-281 MR2467853

[25] J Voight, Tables of totally real number fields, tables of calculations (2012) Available at http://www.math.dartmouth.edu/ jvoight/nf-tables/index.html

[26] L C Washington, Introduction to cyclotomic fields, 2nd edition, Graduate Texts in Math. 83, Springer, New York (1997) MR1421575

[27] R Zimmert, Ideale kleiner Norm in Idealklassen und eine Regulatorabschätzung, Invent. Math. 62 (1981) 367-380 MR604833

Institut für Mathematik, Johann Wolfgang Goethe Universität Robert-Mayer-Str. 6-8, D-60325 Frankfurt am Main, Germany dzambic@math.uni-frankfurt.de

Proposed: Walter Neumann

Seconded: Ronald Stern, Simon Donaldson
Received: 31 January 2014

Revised: 4 July 2014 